Voix et Images

voixetimages

\title{
Anne Hébert : « Un bruit de soie »
}

\section{Gilles Marcotte}

Volume 24, numéro 2 (71), hiver 1999

Poésie québécoise et histoire littéraire

URI : https://id.erudit.org/iderudit/201429ar

DOI : https://doi.org/10.7202/201429ar

Aller au sommaire du numéro

\section{Éditeur(s)}

Université du Québec à Montréal

\section{ISSN}

0318-9201 (imprimé)

1705-933X (numérique)

Découvrir la revue

\section{Citer cet article}

Marcotte, G. (1999). Anne Hébert : « Un bruit de soie ». Voix et Images, 24(2), 301-309. https://doi.org/10.7202/201429ar

\section{Résumé de l'article}

Gilles Marcotte propose ici une explication de texte de l'avant-dernier poème du Tombeau des rois d'Anne Hébert, poème peu souvent cité, négligé par les anthologies, qui lui semble résister à la lecture eschatologique généralement reçue de ce recueil. d'utilisation que vous pouvez consulter en ligne.

https://apropos.erudit.org/fr/usagers/politique-dutilisation/ 


\section{Anne Hébert: «Un bruit de soie»}

\section{Gilles Marcotte, Université de Montréal}

Gilles Marcotte propose ici une explication de texte de l'avant-dernier poème du Tombeau des rois d'Anne Hébert, poème peu souvent cité, négligé par les anthologies, qui lui semble résister à la lecture eschatologique généralement reçue de ce recueil.

\section{Un bruit de soie}

Un bruit de soie plus lisse que le vent Passage de la lumière sur un paysage d'eau.

L'éclat de midi efface ta forme devant moi

Tu trembles et luis comme un miroir

Tu m'offres le soleil à boire

À même ton visage absent.

Trop de lumière empêche de voir;

l'un et l'autre torche blanche,

grand vide de midi

Se chercher à travers le feu et l'eau

fumée.

Les espèces du monde sont réduites à deux

$\mathrm{Ni}$ bêtes ni fleurs ni nuages.

Sous les cils une lueur de braise chante à tue-tête.

Nos bras étendus nous précèdent de deux pas

Serviteurs avides et étonnés

En cette dense forêt de la chaleur déployée.

Lente traversée.

Aveugle je reconnais sous mon ongle

la pure colonne de ton coeur dressé

Sa douceur que j'invente pour dormir

Je l'imagine si juste que je défaille.

Mes mains écartent le jour comme un rideau

L'ombre d'un seul arbre étale la nuit à nos pieds

Et découvre cette calme immobile distance

Entre tes doigts de sable et mes paumes toutes fleuries. 
Le poème dont j'ai choisi de parler n'est pas un des plus importants, des plus célèbres d'Anne Hébert. C'est même, par excellence, le poème du Tombeau des rois dont on se souvient le moins, j'en ai fait la vérification auprès de quelques très bons lecteurs. Je ne le trouve dans aucune anthologie, sauf celle qui accompagne la monographie de René Lacôte, parue dans la collection des "Poètes d'aujourd'hui" en $1969^{1}$. Ce n'est pas, j'imagine, qu'on le trouve médiocre, ou indifférent. Mais il n'impose pas à l'esprit une image-choc, comme "La fille maigre" ou "Le tombeau des rois", dans le recueil qui emprunte son titre à ce dernier poème. Il semble même échapper au mouvement dramatique qui porte l'ensemble du recueil, depuis les images hésitantes du commencement jusqu'à celles, absolument foudroyantes, de la fin. Coincé entre un poème particulièrement intense, "Paysage", où la révolte atteint à l'intensité de la rage: "Roulée dans ma rage / Comme dans un manteau galeux", et le poème du "Tombeau des rois", il semble n'entretenir avec l'un ou avec l'autre que des relations assez faibles. La révolte en est absente, et rien, semblet-il, dans "Un bruit de soie ${ }^{2}$, ne laisse soupçonner, ne prépare la grande résolution du "Tombeau".

C'est le hasard, celui d'un choix de textes pour un enseignement de première année, qui m'a conduit à ce poème. Mais le hasard n'est pas fait que de hasard, et je concède volontiers que "Un bruit de soie" m'a d'abord attiré, justement parce qu'il ne jouait pas un rôle précis, un rôle attendu dans l'organisation du recueil, parce qu'il semblait faire difficulté par rapport à la lecture eschatologique qu'on fait le plus souvent du recueil d'Anne Hébert, lecture que j'ai moi-même propagée pendant un certain nombre d'années. Le choix de ce poème a donc un sens légèrement polémique.

Je procéderai le plus simplement du monde, en me souvenant de l'atmosphère détendue, propice au dialogue, des "Travaux pratiques" de première année, du temps où j'enseignais à l'Université de Montréal. À cette fin, je me suis pourvu d'une question à la fois limpide et redoutable: "Qu'est-ce que ce poème? ", à laquelle je donnerai plus d'une réponise.

Première réponse: "Un bruit de soie" est un récit, ou plutôt une scène érotique. Il importe, à mon avis, de reconnaître d'emblée que ce poème, au contraire de la plupart de ceux qui sont inclus dans Le tombeau des rois, et qui racontent des histoires un peu fantastiques, obéissant à la logique du rêve plutôt qu'à celle des événements quotidiens, raconte une histoire tout à fait vraisemblable, dont on imagine qu'on pourrait la vivre soi-même - avec un peu de chance! Le décor: un "paysage d'eau", dit le poème, c'est-à-dire, peut-être, un lac, sur lequel tombe la lumière

1. René Lacôte, Anne Hébert, Paris, Seghers, coll. "Poètes d'aujourd'hui ", 1969.

2. Anne Hébert, Poèmes, Paris, Seuil, 1960, p. 57-58. 
violente de midi. Dans ce décor, un homme et une femme, la femme narratrice et un amoureux ou un amant auquel elle dit "tu". L'effet de la lumière de midi sur l'eau est de faire trembler toute image qui s'y reflète, et plus particulièrement l'image de l'amant, de la brouiller, voire de la faire disparaître. Parlons d'un éblouissement, où les amants - je retiens ce mot de préférence à celui d'amoureux, à cause de la charge érotique qui sera bientôt évidente —, sous l'effet des éléments combinés, d'un moment de grâce toute physique, ne se reconnaissent vraiment plus euxmêmes, ne se reconnaissent plus l'un l'autre, "l'un et l'autre torche blanche ", et par là même sont violemment attirés l'un vers l'autre. Que l'abondance des signes naturels, leur très forte prégnance poétique, ne nous abuse pas: il s'agit bien ici d'amants, de personnes, comme le suggérait d'ailleurs fortement le titre du poème, repris au début du premier vers. Cette "soie" qui fait du bruit - on est tenté de dire qu'elle bruit (du verbe bruire) ou, à l'imparfait, bruissait, pour donner une assonance à "soie" - a tout du vêtement caressant, séduisant. Claudel, dans son Journal: "La soie, le bruit d'une femme à côté de nous qui marche ${ }^{3}$..." À la première lecture, j'avais cru, sur la foi d'une impression rapide, que le bruit du vent était comparé à celui de la soie - comparaison juste, dirait Lautréamont, image tout à fait convenable. Mais, en réalité, c'est bien la soie du vêtement féminin qui a préséance, qui est le comparé, et le vent, l'élément naturel, qui joue le rôle mineur du comparant.

C'est donc ici le moment de grâce, l'instant absolu; celui de la perte de soi et de l'autre, qui est la marque du coup de foudre. "Les amoureux sont seuls au monde", disait une chanson d'autrefois; et le poème: "Les espèces du monde sont réduites à deux." Les autres espèces, les naturelles, sont disparues: "Ni bêtes ni fleurs ni nuages"; ne restent que deux êtres, une femme et un homme, face à face, avec, "Sous les cils une lueur de braise [qui] chante à tue-tête". Je ne connais pas, dans la littérature québécoise, de scène où soit évoquée de façon si concrète, si poignante, la foudre amoureuse.

Mais ni le poème ni les amants ne peuvent en rester là. L'instant absolu de la passion, qui est l'instant de la perte de soi, de l'absence à soi, par là même ouvre une histoire temporelle, cette recherche "à travers le feu et l'eau / fumée" dont parle le texte. J'insiste: je ne parle pas ici d'une analyse abstraite de l'aventure amoureuse, enfouie dans la sombre forêt des symboles, mais d'une histoire très simple, évidente, éminemment concrète. Les deux amants iront donc, après cet éblouissement, à la recherche l'un de l'autre; une recherche encore marquée par l'hébétement initial, le corps même précédant la prise de conscience: "Nos bras étendus nous précèdent de deux pas". La recherche sera dirigée par les

3. Paul Claudel, Journal, tome 1 (1904-1932), Paris, Gallimard, coll. "Bibliothèque de la Pléiade", 1968, p. 180. 
bras, "serviteurs avides et étonnés", puis par les mains, les doigts, par le toucher. Le regard, la vue n'ont rien à faire ici, les amants agissent sous l'empire d'un désir proprement aveugle, fort de cet aveuglement même. Car, de l'empire du jour nous sommes passés à celui de la nuit, du coup de foudre de midi à ce qu'il faut bien appeler une nuit d'amour. Coup de foudre, nuit d'amour, ce sont bien là des clichés; mais la poésie fait-elle jamais autre chose que de réactiver des clichés, de les faire. rentrer de force, pour ainsi dire, dans l'immédiat de l'expérience? Désormais, le toucher seul s'active: "je reconnais sous mon ongle / la pure colonne de ton cœur dressé", "Mes mains écartent le jour", jusqu'à la rencontre finale, en apothéose, des "doigts de sable" et des "paumes toutes fleuries". Je dis rencontre, le poème parle de "distance". La fusion était l'affaire du coup de foudre; le dialogue amoureux, au contraire, tient les amants à une certaine distance l'un de l'autre, chacun s'étant retrouvé, dans et par l'amour, face à l'autre.

Que cette aventure ait eu lieu, ou non, dans ce qu'on appelle la réalité, ne nous importe évidemment pas. Telle qu'elle nous est racontée ici, elle atteint un niveau de généralité, d'exemplarité qui l'arrache au vécu singulier sans le sacrifier. L'important, pour moi, pour le lecteur que je suis, est qu'elle se présente comme vraisemblable. Il va sans dire, toutefois, que, pour raconter cette histoire, j'ai dû amputer le poème d'un certain nombre d'aspects qu'on peut juger plus importants que la simple histoire d'amour. D'où la deuxième réponse à la question posée: "Un bruit de soie " est une histoire cosmique, une histoire de monde. Cette deuxième histoire relève évidemment de ce que Gaston Bachelard appelle l'«imagination matérielle»; je ferai comme si les éléments matériels avaient, par rapport au récit que je viens de dégager, une existence relativement autonome.

L'histoire du poème, l'histoire racontée par le poème est, ainsi, celle de la lumière et de l'eau: "Passage de la lumière sur un paysage d'eau." Insistons sur la préposition "sur" - tout en notant, au passage, la suite d'allitérations: "soie", "lisse", "passage", "sur", "paysage", toute de douceur - cette préposition qui, entre la lumière et l'eau, établit un rapport de survol, de contact léger. Ce rapport, à la strophe suivante, se fera plus intime, et l'on oserait dire plus inquiétant: "Tu m'offres le soleil à boire", dit le poète, transformant le cliché "la mer à boire". L'union des contraires, du feu et de l'eau, se fait explosive: si le soleil peut être bu, c'est qu'il èst lui-même, contre toute logique, devenu liquide. Les éléments mêmes, à l'instar des amants, perdent la tête, perdent ce qui fait leur nature propre. Cette confusion culmine dans l'étrange, la splendide image de la troisième strophe: "l'un et l'autre torche blanche". L'eau (blanche) est devenue feu, le feu est devenu blanc. On ne saurait aller plus loin dans cette direction, dans cette fusion, et c'est pourquoi, dans la même strophe, s'amorce, sous le signe de la quête, de la recherche ("Se cher- 
cher", dit le poème), une démarche plus lente ("Lente traversée", dira tout à l'heure le poème), où le paradoxe insoutenable voudra trouver sa résolution. L'étonnante apparition du mot "fumée", constituant à lui seul un vers à la fin de la strophe:

Se chercher à travers le feu et l'eau

fumée

annonce déjà une tentative de composition, la fumée n'étant pas un élément pur, mais le produit (impur) de l'eau et du feu.

Cette tentative, toutefois, ne se poursuivra qu'après deux vers sur lesquels je suis passé un peu rapidement tout à l'heure et qui sont, pour moi, les plus énigmatiques du poème:

Les espèces du monde sont réduites à deux

Ni bêtes ni fleurs ni nuages.

Il s'agit donc bien du monde, ici; d'un monde privé de ses espèces animales, mais aussi des fleurs, des nuages, en somme de tout ce qui en fait un jardin, un lieu animé, mouvant, divers. Mais que sont donc les deux "espèces" résiduelles? Dirons-nous, pour rester dans le domaine cosmique, que ce sont l'eau et le feu, si puissamment évoqués dans les strophes précédentes? Ou encore, avec plus de vraisemblance, les amants eux-mêmes? Il me paraît que le mot "espèces" doit être reçu dans un sens assez large et qu'il a surtout pour fonction de marquer le caractère en quelque sorte élémentaire, premier, de ce qui se passe dans le poème. "Il les créa homme et femme", dit la Bible, et l'expression "deux espèces" peut également évoquer le rite sacramentaire de la transsubstantiation (le Christ est présent sous les deux espèces, le pain et le vin). Mais je ne m'enfoncerai pas dans les dédales de ces possibles allusions. J'en reste aux "espèces du monde" dont parle le poète, je m'enchante de la multiplicité des sens possibles et je m'attache au nombre, au nombre "deux".

Tout s'est passé, depuis le début du poème, sous le signe du "deux", de la dualité: on pensera d'abord, évidemment, aux deux amants. Mais cette dualité ne sera véritablement posée comme telle que dans la deuxième partie, à partir des espèces du monde "réduites à deux". Elle insistera, encore, au début de la strophe suivante: "Nos bras étendus nous précèdent de deux pas", et elle sera d'autant plus visible qu'un autre chiffre, l'autre chiffre, le "un", fera son apparition, de façon allusive d'abord, dans "la pure colonne de ton cour dressé", puis, explicitement, dans un vers de la dernière strophe: "L'ombre d'un seul arbre étale la nuit à nos pieds". C'est dire que, dans la forêt où se trouvent maintenant les amants - ils ont changé complètement de paysage, du midi liquide, violence de l'absolu, ils sont passés à la nuit favorable, la forestière -, l'unité et la dualité ne s'opposent pas, ne constituent pas des postulations contradictoires. Au contraire, c'est grâce à "un seul arbre", à l'arbre unique (comment éviterions-nous de penser à la Genèse?) que la dualité pourra 
recueillir, dans le dernier vers, toutes les grâces naturelles qu'elle avait elle-même exclues: "tes doigts de sable et mes paumes toutes fleuries". Dans cette dernière image, il n'est plus question de fusion, on l'aura remarqué; les deux "espèces" demeurent parfaitement distinctes, et l'unité du couple amoureux ne se fait plus au détriment de la dualité. Une loi s'est imposée au cours du poème, celle de la distance: "cette calme immobile distance" dont parle l'avant-dernier vers. Cette loi n'est pas uniquement celle du couple humain, de deux personnes singulières, dont les belles images naturelles du poème seraient le commentaire, l'ornement. Elle s'impose dans tous les règnes du monde. Ce poème-ci, "Un bruit de soie", parle du monde, parle pour le monde; et je dirais "pour.le salut du monde", en me souvenant d'une parole de la messe dominicale ("pour la gloire de Dieu et le salut du monde").

Un poème, disions-nous. Il faut ajouter: de toute évidence, un poème d'Anne Hébert. Et telle est la troisième réponse que je donne à ma question: "Un bruit de soie" est un poème d'Anne Hébert. C'est là une affirmation apparemment vaine, puisque le poème est signé de ce nom. J'espère lui donner une signification un peu plus substantielle. Les images, les mots que nous lisons dans "Un bruit de soie", nous les reconnaissons véritablement parce que nous les avons déjà rencontrés dans plusieurs poèmes du Tombeau des rois. Nous ne lisons pas le mot "monde", dans ce texte, comme nous le lirions dans un autre ensemble poétique; aussitôt notre oreille, notre imagination nous le fait retrouver comme un être familier, doté pour ainsi dire d'une personnalité propre. Le monde dont il est ici question, c'est bien celui du beau vers de "La chambre de bois": "La place du monde flambe comme une forge", c'est celui encore dont parle le poète lorsqu'elle évoque les "chemins du monde", ou encore "la beauté du monde", et je ne cite pas toutes les occurrences. Il y a toujours, dans la poésie d'Anne Hébert, cet appel d'un espace plus vaste, un espace qui, on ne l'a peut-être pas assez vu, déborde les stigmates purement individuels. Le "paysage d'eau " du premier vers, nous ne le rencontrons pas non plus pour la première fois, de même que la "dense forêt", qui renvoie directement aux deux vers d'"Éveil au seuil d'une fontaine": "Dense forêt / Des songes inattendus". Et encore, ces mains "qui écartent le jour comme un rideau", ce sont bien les mêmes que le poète, quelques pages plus haut, avait l'idée de "planter [...] au jardin". Les ressemblances, les rappels foisonnent dans notre lecture de "Un bruit de soie", nous sommes bien toujours chez Anne Hébert, dans le paysage verbal qu'Anne Hébert ne cesse de travailler. Mais il devient urgent de souligner que les mêmes mots, les mêmes images n'ont pas toujours, de poème en poème, la même signification, la même portée. Ils ressemblent à des êtres vivants, qui bougent, se modifient, et il faut prendre garde de ne pas les figer dans une seule attitude, un seul rôle.

Je disais, au début de ce texte, que "Un bruit de soie " entretient des relations assez faibles avec le poème qui le suit dans le recueil, "Le tom- 
beau des rois". C'était là une affirmation fondée sur une lecture trop rapide des poèmes. Les "Serviteurs avides et étonnés" de notre poème ne peuvent pas ne pas nous faire penser à la narratrice "Étonnée / À peine née" du poème suivant. De même, la correspondance s'établit aussitôt entre le syntagme "ton visage absent", qu'on lit dans "Un bruit de soie", et l'expression "ma face absente" du poème suivant :

Le masque d'or sur ma face absente

Des fleurs violettes en guise de prunelles,

L'ombre de l'amour me maquille à petits traits précis.

Mais "visage" n'est point "face", et l'absence, dans l'un et l'autre vers, n'a pas le même sens. L'absence du visage dans "Un bruit de soie" est l'effet d'un éblouissement amoureux et ne saurait être limitée au sens d'une privation; elle est une grâce excessive, mais une grâce, alors que la "face absente" du "Tombeau des rois" parle d'une aliénation totale, d'une soumission atroce à l'appétit d'autrui. Une différence semblable se marque entre le vers de la troisième strophe: "Trop de lumière empêche de voir", et la conclusion tant de fois commentée de l'autre poème:

D'où vient donc que cet oiseau frémit

Et tourne vers le matin

Ses prunelles crevées?

À la maxime énoncée dans "Un bruit de soie", et qui a presque la force d'une injonction, le premier poème obéit en écartant "le jour comme un rideau ": résolution douce. Dans "Le tombeau des rois", au contraire, les enjeux sont présentés de façon si dramatique, si intense, que c'est la source même du regard qui doit être détruite pour permettre l'accès au "matin ", à la vie, peut-être à l'amour.

La même parenté et les mêmes différences, enfin, apparaissent dans ce qui identifie le plus sûrement les poèmes d'Anne Hébert, la diction. Cette diction très particulière, qui refuse de se faire remarquer - Pierre Emmanuel parle, en ce sens, d'un "verbe austère et sec, rompu, soigneusement exclu de la musique ${ }^{4}$-, qui range les mots les plus simples, les plus courants dans des phrases assez courtes, souvent non verbales, ressemblant à des constats, mais par leur pauvreté apparente même produisant un effet d'enchantement. Tout paraît égal, uni, à peine modulé, et pourtant les ruptures sont nombreuses - par exemple lorsque, après une phrase verbale apparemment complète, la suivante commence par un infinitif d'action: "Se chercher à travers le feu et l'eau / fumée". Ces ruptures sont moins sensibles dans "Un bruit de soie" que dans "Le tombeau des rois", parce que les vers y sont plus largement découpés, mais c'est bien la même musique, ici et là, qui se fait entendre, la très puissante, l'insidieuse musique de la discrétion et de la justesse. La formule de

4. Pierre Emmanuel, "Présentation", Poèmes, op. cit., p. 11. 
l'enchantement est donnée, dans notre poème, par deux vers saisissants de l'avant-dernière strophe:

Sa douceur que j'invente pour dormir

Je l'imagine si juste que je défaille.

Nulle part ailleurs Anne Hébert n'a défini plus justement son mode poétique, et ce n'est pas pour rien qu'elle utilise ici les verbes de l'action créatrice: "j'invente", "j'imagine". Son poème doit tout à la recherche impitoyable de la justesse, qui n'est pas seulement l'arrimage exact du mot à ce qu'il exprime, ou des mots entre eux dans une syntaxe admirablement économe, mais ce qui amène le langage aux portes de quelque chose qu'il provoque et qui le dépasse: d'où la défaillance dont parle le poème. Je ne puis me résoudre à penser qu'il ne s'agirait ici que d'une simple extase sentimentale. Le poète Joseph Brodsky, qu'on ne saurait suspecter de complaisance à l'égard des spiritualités vagues, a écrit que "the infinite is poetry's standard turf ${ }^{5}$ " (l'infini est le terrain normal de la poésie). Il ne définit pas cet infini. Je ne le ferai pas non plus.

"Un bruit de soie", c'est donc tout ça: une histoire, une scène érotique; une histoire cosmique, une histoire des éléments du monde; un poème d'Anne Hébert. C'est aussi, sans autre détermination, un poème, c'est-à-dire une histoire de mots, une histoire de langage, l'expression par le langage d'une fidélité profonde à l'égard de lui-même, de ce qu'il porte. De phrase en phrase, ou plus justement de vers en vers, il nous fait éprouver le risque toujours nouveau de l'action de parler. J'insiste, aussi, sur l'article: un poème. Ce poème n'a pas besoin des autres pour être lu. Il constitue un organisme complet, suffisant, que chaque lecteur est invité à investir avec ses propres attentes, ses propres demandes. Je ne regrette pas de l'avoir soumis aux manœuvres habituelles de l'explication de texte, mais je le relirai en cherchant à retrouver, enrichi, l'enthousiasme qui m'avait saisi à la première lecture.

\section{Post-scriptum}

L'explication de texte qui précède peut, à juste titre, être interprétée comme un acte de résistance à l'égard de la thématique même du colloque auquel il était destiné. Il m'est apparu, en effet, comme je l'indique plus haut, que le poème intitulé "Un bruit de soie" entrait difficilement soyons franc: n'entrait pas du tout - dans le récit psychosocial où l'on fait entrer d'habitude la poésie d'Anne Hébert. Celle-ci, selon le récit consacré, constituerait à la fois une exploration extrêmement profonde, audacieuse, d'une conscience collective marquée par le malheur, l'aliénation, et une sortie de ce malheur, sortie dont témoigneraient les poèmes à

5. Joseph Brodsky, On Grief and Reason, New York, The Noonday Press, 1997, p. 322. C'est moi qui traduis. 
larges versets de "Naissance de la parole". Or, "Un bruit de soie", non seulement ne participe pas à ce mouvement de libération, à ce passage, puisqu'il pose la passion amoureuse et la passion du monde dans un bic et nunc indubitable, mais il invite à lire les poèmes qui l'entourent à sa propre lumière, celle d'une positivité qui s'accomplit dans le temps même du poème, fût-ce contre sa thématique explicite. Cela revient à dire que les poèmes du Tombeau des rois, lus souvent comme l'exploration d'un malheur qui serait surmonté dans "Naissance de la parole", accomplissent un sens (j'oserais dire un bonheur) qui prend en charge, si l'on veut, les malheurs d'un temps (et c'est pourquoi je ne puis récuser totalement la lecture habituelle), mais les transforment aussitôt, sans attendre, en résistance à ces malheurs. Je refuserai désormais, par exemple, de lire "La chambre fermée" ou "L'envers du monde" comme des récits de malheur qui s'aboliraient pour ainsi dire dans la poésie ultérieure d'Anne Hébert. Le monde, le sens ne se donnent pas avec moins de faveur dans ces poèmes que dans "Un bruit de soie".

Est-ce à dire qu'il soit impossible de réunir dans un seul propos la poésie et l'histoire, particulièrement cette histoire littéraire dont, au reste, on ne connaît pas trop exactement le statut et les outils, et qui arrive si difficilement à se distinguer de l'histoire sans adjectif? Je me garde bien de l'affirmer. Mais je rêve d'une histoire de la poésie qui serait aussi une histoire de ses résistances, de ses écarts, qui reconnaîtrait en elle les formes diverses d'une "contradiction" toujours agissante, selon le titre du grand ouvrage de Pierre Popovic ${ }^{6}$.

6. Pierre Popovic, La contradiction du poème. Poésie et discours social au Québec de 1948 à 1953, Candiac, Balzac, coll. "L'univers des discours", 1992. 